\title{
Household survey of availability of long-lasting insecticide-treated nets and its determinants in rural Mozambique
}

\author{
Inocencio M Quive ${ }^{1 *}$, Baltazar Candrinho ${ }^{1}$ and Diederike Geelhoed ${ }^{2}$
}

\begin{abstract}
Background: Mosquito nets treated with long-lasting insecticide offer highly effective personal protection against malaria transmission. In Mozambique, nets are distributed freely in antenatal care visits since 2006 and through mass distribution campaigns since 2009, but the country has not yet been able to report a consistent decline in malaria incidence. Routine data show that Changara District, Tete Province, shows an increase in malaria cases, although it has a reasonable theoretical coverage of nets. This study evaluated household availability of nets and its determinants in Changara district.
\end{abstract}

Methodology: Quantitative household survey at the end of 2013, in a representative sample of 450 households in 30 villages of Changara district, using the sampling method of randomly selected households in clusters selected with probability proportional to size. Data were analysed with Epi-Info version 7.1.2.0. The significance level was 0. 05.

Results: Of 450 households, $62.5 \%$ (95\% Cl 57.5-66.7) had at least one long-lasting insecticide-treated net. Availability of nets showed a positive association with socioeconomic status and the existence of at least one pregnant woman or child under 5 years in the household, but a negative association with distance between health facility and residence. Most of the observed nets were not in good condition, only $19.2 \%(95 \% \mathrm{Cl} 15.7-23.2)$ of households had at least one net in good condition. The condition of the nets reduced with increasing number of washes.

Conclusions: The household availability of long-lasting insecticide-treated nets in Changara district has not yet reached levels that may have an impact on the incidence of malaria, despite distribution through campaign and antenatal care. The habit of washing nets frequently reduces their lifespan. It is recommended to strengthen education on good practices of net conservation, in addition to their distribution.

Keywords: Long-lasting insecticide-treated nets, Malaria prevention, Mozambique

\section{Background}

Consistent and correct use of long-lasting insecticidetreated nets (LLITN) does reduce malaria incidence in under fives by up to $50 \%$, incidence of severe malaria by $45 \%$, and infant mortality by $25 \%$ [1-4]. Universal coverage of LLITNs is defined as the use of a net by more than $80 \%$ of the population at risk, counting one net for each two persons [5]. Consensus in the malaria Vector Control Working Group (VCWG) indicates that universal

\footnotetext{
*Correspondence: Inocencio_marcos@yahoo.com.br

${ }^{1}$ Tete Provincial Health Directorate, Tete City, Mozambique

Full list of author information is available at the end of the article
}

coverage, reached through mass distribution campaigns, is the main strategy for the rapid attainment of malaria control [4]. Mozambique, a malaria endemic country, has introduced mass distribution campaigns every 3-4 years in selected districts since 2009, in addition to distribution through antenatal care. However, the country is not yet able to report a consistent decline in malaria incidence $[6,7]$.

The physical integrity of a net is important for its level of protection against malaria [8]. Studies on the conservation of nets suggest that considerable damage frequently occurs already within 1 year, related to poor maintenance. This damage increases with duration of use. 
Many nets do not last for more than 2 years, although some have stated that lifespan of nets can be up to 4 years $[1,2,9-12]$.

Tete Province, in central Mozambique, is one of the areas showing a recent increase in malaria incidence, despite the distribution of a considerable number of nets since 2010. Changara is the district in Tete Province which, theoretically, based on net distribution numbers relative to the population, should have the highest net coverage in the province, close to universal, assuming an effective lifespan of around 3-4 years. Nevertheless, although malaria incidence per 1,000 population was 34 in 2010 (when the mass distribution was done), routine data show an increasing incidence of malaria since then, to 64 in 2013. If the actual net coverage in the district would be lower than expected, or the physical integrity of the nets compromised, this might explain the apparent contradiction between expected net coverage and reported malaria incidence.

This study evaluated coverage and conservation status of LLITN in Changara district, to shed light on the apparent contradiction between expected net coverage and reported malaria incidence.

\section{Methods}

A household survey was implemented in Changara district, Tete province, Mozambique, with data collection in December 2013, during the rainy season. The district was purposely selected, based on its apparent contradiction between expected net coverage and reported malaria incidence. The public health system in Changara district consists of 13 outpatient units and one inpatient unit serving a population of 189,000 [13], indicating a low ratio of health care facilities per inhabitant $(1 / 13,400)$. The only mass LLITN distribution campaign in the district was in 2011, but distribution through antenatal care is on-going. The district has a semi-arid climate with strong seasonal differences in rainfall, and its inhabitants are mainly subsistence farmers [14]. A modified cluster sample survey $(30 \times 15)$ was employed [15]. The sample was obtained in two steps. First, 30 conglomerates were randomly selected, proportional to their population size. Then, 15 households per conglomerate were randomly selected (systematically), totaling 450 households in the sample. The questionnaire, applied to one representative from each household, was based on previous questionnaires of similar surveys in Mozambique.

The study variables were classified in two categories:

Socio-demographic details Age, gender and educational level of household representative, number of pregnant women and children under 5 years of age in household, total number of people in the household, construction material of the walls and roof of the household house, and household ownership of assets (livestock);

Characteristics related to LLITN Presence of nets in the household, source of each net (free distribution through campaign or prenatal consultation by non-governmental organizations, commercial purchase), time passed since reception of each net (net age), laundry habits of nets (number of times washed), physical integrity of each net (number of holes with a diameter greater than $1 \mathrm{~cm}$ ).

The questionnaire was applied by one of five trained research assistants, under supervision of two principal investigators, with support from a community representative in each conglomerate, who assisted with translation from Portuguese into the local language. Quality of data collection was ensured during daily meetings of the entire team during the field work.

The statistical package EPI-Info v 7.1.2.0 was used to digitalize, verify, and analyse data. Frequencies were calculated for all variables with their confidence intervals. Educational level was combined with house construction material and ownership of assets into a new variable indicating the socioeconomic status of each household. Chi square tests were used to detect statistically significant differences between groups, with a significance level of 0.05 .

The study was implemented within routine activities of the provincial malaria control programme, with authorization from the Provincial Health Authorities, District Government and local community leaders. Written informed consent was obtained from each participating household representative.

\section{Results}

During data collection, 30 villages were visited and 450 household representatives interviewed, 15 households per village. No household was excluded, and no representatives denied participation. The socio-demographic characteristics of the participants are summarized in Table 1.

Results regarding household availability and physical integrity of LLITN are summarized in Table 2. Among the participating households, $62.5 \%$ were found to have at least one LLITN, but only $19.2 \%$ of households were found to have at least one LLITN with good physical integrity. The mean number of persons per household was 5.8 (SD 3.0), however, the mean number of LLITN in any condition per household was only 1.1 (SD 1.2), and the mean number of LLITN with good physical integrity was just 0.3 (SD 0.6). Even in households with at least one LLITN in good condition, the mean number of LLITN in good condition was low (1.4, SD 0.7) compared to the mean number of persons (6.5, SD 2.9). Overall, most of 
Table 1 Socio-demographic characteristics of the participants

\begin{tabular}{|c|c|c|c|c|}
\hline Variable & Categories & $\mathbf{N}$ & $\%$ & $95 \% \mathrm{Cl}$ \\
\hline \multirow[t]{2}{*}{ Gender } & Female & 287 & 63.8 & $59.1-68.2$ \\
\hline & Male & 163 & 36.2 & $31.8-40.9$ \\
\hline \multirow[t]{2}{*}{ Representative } & Household head & 223 & 49.6 & $44.9-54.3$ \\
\hline & Other & 227 & 50.4 & $45.7-55.2$ \\
\hline \multirow[t]{4}{*}{ Socio-economic level } & Lowest 25\% & 164 & 38.9 & $34.2-43.7$ \\
\hline & Medium-low 25\% & 143 & 33.9 & $29.4-38.7$ \\
\hline & Medium-high 25\% & 100 & 23.7 & $19.8-28.1$ \\
\hline & High 25\% & 15 & 3.6 & $2.1-5.9$ \\
\hline \multirow[t]{2}{*}{ Distance to nearest health facility } & Close to health facility <10 km & 225 & 50.0 & $45.2-54.8$ \\
\hline & Far from health facility $>10 \mathrm{~km}$ & 225 & 50.0 & $45.2-54.8$ \\
\hline \multirow[t]{4}{*}{ Household members } & Total number in all households & 2,612 & 100 & - \\
\hline & Mean number per household & \multicolumn{3}{|c|}{5.8 (SD 3.0); range 1-18 } \\
\hline & Number of households with at least one pregnant women & 37 & 8.2 & $5.9-11.3$ \\
\hline & Number of households with at least one under five & 300 & 66.7 & $62.1-71.0$ \\
\hline
\end{tabular}

Table 2 Household availability LLITN

\begin{tabular}{|c|c|c|c|c|}
\hline Variable & Categories & $\mathbf{N}$ & $\%$ & $95 \% \mathrm{Cl}$ \\
\hline \multirow[t]{4}{*}{ Availability of LLITN } & Number of households with at least one LLITN (regardless of its condition) & 280 & 62.5 & $57.8-67.0$ \\
\hline & Number of households without any LLITN (regardless of condition) & 168 & 37.5 & $33.0-42.2$ \\
\hline & Number of households with at least one LLITN (in good condition) & 86 & 19.2 & $15.7-23.2$ \\
\hline & Number of households without any LLITN (in good condition) & 362 & 80.8 & $76.8-84.3$ \\
\hline
\end{tabular}

the nets $(75.5 \%$; 95\% CI 69.5-80.0) originated from the 2011 mass distribution campaign, followed by antenatal care distribution (16.5\%; 95\% CI 10.7-24.0).

Determinants of household availability of at least one LLITN (regardless of its condition) included socioeconomic status, where households with a lower status were less likely to have at least one LLITN, than those with a higher status (OR 0.6; 95\% CI 0.4-0.9; $\mathrm{p}=0.02$ ). Distance to the nearest health facility also determined household LLITN availability: those living more than $10 \mathrm{~km}$ from a health facility were less likely to have at least one LLITN, than households who lived closer (OR 0.6, 95\% CI 0.4$0.9 ; \mathrm{p}=0.006)$. In addition, the existence of a pregnant woman or under five was associated with household LLITN availability: households with at least one pregnant woman or under five were more likely to have at least one LLITN than households without pregnant woman or under five (OR 1.5; 95\% CI 1.0-2.2; $\mathrm{p}=0.03$ ).

In those households with at least one LLITN, availability of at least one net with good physical integrity was associated again with socioeconomic status, where households with a lower status were less likely to have at least one LLITN, than those with a higher status (OR 0.5; $95 \%$ CI $0.3-0.9 ; \mathrm{p}=0.08)$. It was also determined by area of residence: households living in the least accessible part of the district were less likely to have at least one good net, than households who lived elsewhere (OR 0.5, 95\% CI $0.3-0.8 ; \mathrm{p}=0.004)$. In addition, it was associated with the existence of pregnant women or under fives in the household: households with at least one pregnant woman or under five were more likely to have at least one good LLITN than households without pregnant woman under five (OR 1.8; 95\% CI 1.0-3.3; $\mathrm{p}=0.02$ ). Finally, there was an association with washing practices: those households which already had washed their LLITN four times or more were less likely to have at least one LLITN in good condition that the households which had washed less often (OR 0.5; 95\% CI 0.3-0.8; p = 0.002).

\section{Discussion}

This survey in Changara District raises important issues for local malaria control with LLITN. The WHO and the Roll-Back Malaria Initiative recommend 100\% universal coverage with LLITN to long-term high-risk populations (counting one net for each two persons) with a target of at least $80 \%$ utilization [5]. The results showed a high percentage of households without any LLITN, and an insufficient mean number of LLITN with good 
physical integrity as compared to the mean number of persons per household. Even in households which had at least one LLITN with physical integrity, the mean number of LLITN was inadequate to protect all household members. Therefore, the current LLITN availability in Changara is far from ideal, which probably explains the high malaria incidence locally.

The study results suggest that low LLITN availability is related to the limited effective lifespan of the LLITN, mainly due to inappropriate washing practices. Instead of a potential lifespan of up to 4 years, the local LLITN seem to keep their integrity during fewer years, as reported in others studies $[16,17]$. Poor maintenance of nets and inappropriate washing practices have also been reported by other studies, which suggest that the usefulness of the net decreases rapidly through physical damage and reduction of the effect of the insecticide related to washing, amongst other factors $[18,19]$. This study suggests the need to extend the lifetime of the LLITN through education programmes in the community on appropriate ways of handling, washing and repair, which has been shown feasible and effective elsewhere [20].

The results show that many LLITN were provided through universal distribution campaign, and relatively few through distribution in antenatal care, although households with pregnant women or under fives were more likely to possess a LLITN. This indicates an insufficient capacity of antenatal care distribution to provide initial LLITN and their timely replacement, once no longer in good condition, also observed in other studies [21]. This suggests the need to strengthen the distribution channels, with more frequent campaigns to rapidly increase coverage, and increased distribution through health facilities to maintain elevated rates [22]. As a distribution channel, the universal distribution campaign is highly effective and should be preferred in view of the inequities observed in this study with regard to socioeconomic status and distance to a health facility, despite its major logistical burden [23]. The most disadvantaged groups in a population access health care less easily, and therefore distribution by health facility is inherently inequitable.

There are some limitations to our study. It was difficult to measure consistently and rigorously the physical integrity of the LLITN in the field. Insecticide content of the nets was also not measured. Therefore only LLITN without any visible holes $(>1 \mathrm{~cm})$ were considered as in good condition, which may have been excessively strict. However, there is some evidence that mosquitoes indeed can enter in torn nets [16]. No information on malaria incidence in the study population was gathered, and another limitation is the lack of information on bed net use. However, as so few households had at least one LLITN in good condition, even with optimal usage the protection offered in the population would be limited.

\section{Conclusions}

The LLITN household availability in Changara district has not yet reached levels that may have an impact on the incidence of malaria, despite distribution through campaign and antenatal care. The lifespan of the LLITN appears to be much shorter than previously assumed, related to inappropriate washing practices. Some level of inequity in LLITN availability was observed associated with distance to health facility, socioeconomic status, and residence in less accessible areas, although households with pregnant women or under fives were more likely to possess a LLITN. Our study suggests a need to increase the frequency of mass distribution campaigns and strengthening routine distribution through health facilities, intensifying education programmes on net maintenance to reduce the LLITN attrition rate, in order to increase household availability and consequently reduce the burden of malaria in rural Mozambique.

\section{Authors' contributions}

IQ participated in the design of the study, collection of data, statistical analysis and drafted the manuscript. BC designed the study, coordenated and supervised data collection, and critically reviewed the manuscript. DG participated in the design, performed the statistical analysis, and helped draft the manuscript. All authors read and approved the final manuscript.

\section{Author details}

${ }^{1}$ Tete Provincial Health Directorate, Tete City, Mozambique. ${ }^{2}$ Danish Ministry of Foreign Affairs, Provincial Directorate of Health, Tete City, Tete Province, Mozambique.

\section{Acknowledgements}

The research was funded by Tete Provincial Health Directorate through budget support from the Royal Danish Embassy. The authors are deeply grateful to all survey participants who gave their time for the study. Special thanks goes to the Changara District Health Services for their support in the data collection.

Compliance with ethical guidelines

Competing interests

The authors declare that they have no competing interests.

Received: 13 March 2015 Accepted: 16 July 2015

Published online: 06 August 2015

\section{References}

1. Fegan GW, Noor AM, Akhwale WS, Cousens S, Snow RW (2007) Effect of expanded insecticide-treated bednet coverage on child survivalin rural Kenya: a longitudinal study. Lancet 370:1035-1039

2. Gimnig JE, Vulule JM, Lo TQ, Kamau L, Kolczak MS, Phillips-Howard PA et al (2003) Impact of permethrin-treated bednets on entomological indices in an area of intense year-round malaria transmission. Am J Trop Med Hyg 68(Suppl 4):16-22

3. Killeen GF, Smith TA, Ferguson HM, Mshinda $H$, Abdulla S, Lengeler $C$ et al (2007) Preventing childhood malaria in Africa by protecting adults from mosquitoes with insecticide-treated nets. PLoS Med 4:e229. doi:10.1371/ journal.pmed.0040229 
4. Roll Back Malaria Partnership (2005) Global strategic plan: roll back malaria 2005-2015. World Health Organization, Geneva

5. Roll Back Malaria Partnership (2008) Global malaria action plan. World Health Organization, Geneva

6. Ministry of Health (2011) National malaria control programme. Annual report 2011. Maputo, Mozambique

7. Ministry of Health (2012) National malaria control programme. Annual report 2012. Maputo, Mozambique

8. WHO (2011) Guidelines for monitoring the durability of long-lasting insecticidal mosquito nets under operational conditions control of neglected tropical diseases. World Health Organization Pesticide Evaluation Scheme and Global Malaria Programme Vector Control Unit

9. Erlanger TE, Enayati AA, Hemingway J, Mshinda H, Tami A, Lengeler C (2004) Field issues related to effectiveness of insecticide-treated nets in Tanzania. Med Vet Entomol 18:153-160

10. Githinji S, Herbst S, Kistemann T, Noor AM (2010) Mosquito nets in a rural area of Western Kenya: ownership, use and quality. Malar J 9:250

11. Kilian A, Byamukama W, Pigeon O, Atieli F, Duchon S, Phan C (2008) Longterm field performance of a polyester-based long-lasting insecticidal net in rural Uganda. Malar J 7:49. doi:10.1186/1475-2875-7-49

12. Protopopoff N, Van Bortel W, Marcotty T, Van Herp M, Maes P, Baza D et al (2007) Spatial targeted vector control in the highlands of Burundi and its impact on malaria transmission. Malar J 6:158. doi:10.1186/1475-2875-6-158

13. National Institute of Statistics (2007) Census results. http://www.tete.gov. mz/informacao/estatistica/resultados_censo.pdf. Accessed 15 Sept 2013

14. Ministry of State Administration (2005) Profile Changara District. http:// www.govnet.gov.mz. Accessed 15 Sept 2013

15. Hoshaw-Woodard S (2001) Description and comparison of the methods of cluster sampling and lot quality assurance sampling to assess immunization coverage. Geneva, World Health Organization. http://whqlibdoc. who.int/hq/2001/who_v\&b_01.26.pdf. Accessed 15 Sept 2013
16. Gnanguenon V, Azondekon R, Oke-Agbo F, Beach R, Akogbeto M (2014) Durability assessment results suggest a serviceable life of two, rather than three, years for the current long-lasting insecticidal (mosquito) net (LLIN) intervention in Benin. BMC Infect Dis 14:69

17. Mejía P, Teklehaimanot HD, Tesfaye Y, Teklehaimanot A (2013) Physical condition of Olyset nets after five years of utilization in rural western Kenya. Malar J 12:158

18. Mutuku FM, Khambira M, Bisanzio D, Mungai P, Mwanzo I, Muchiri EM et al (2013) Physical condition and maintenance of mosquito bed nets in Kwale County, coastal Kenya. Malar J 12:46

19. Batisso E, Habte T, Tesfaye G, Getachew D, Tekalegne A, Kilian A et al (2012) A stitch in time: a cross-sectional survey looking at long lasting insecticide-treated bed net ownership, utilization and attrition in SNNPR, Ethiopia. Malar J 11:183

20. Harvey SA, Olórtegui MP, Leontsini E, Asayag CR, Scott K, Winch PJ (2013) Trials of improved practices (TIPS): a strategy for making long-lasting nets last longer? Am J Trop Med Hyg 88:1109-1115. doi:10.4269/ ajtmh.12-0641

21. Eijk AMV, Hill J, Larsen DA, Webster J, Steketee RW, Eisele TP et al (2013) Coverage of intermittent preventive treatment and insecticide-treated nets for the control of malaria during pregnancy in sub-Saharan Africa: a synthesis and meta-analysis of national survey data, 2009-11. Lancet Infect Dis 13:1029-1042

22. Sexton RA (2011) Best practices for an insecticide-treated bed net distribution programme in sub-Saharan eastern Africa. Malar J 10:157

23. Willey AB, Paintain L, Mangham L, Car J, Schellenberg JA (2012) Strategies for delivering insecticide-treated nets at scale for malaria control: a systematic review. Bull World Health Organ 90:672-684. doi:10.2471/ BLT.11.09477

\section{Submit your next manuscript to BioMed Central and take full advantage of:}

- Convenient online submission

- Thorough peer review

- No space constraints or color figure charges

- Immediate publication on acceptance

- Inclusion in PubMed, CAS, Scopus and Google Scholar

- Research which is freely available for redistribution

Submit your manuscript at

www.biomedcentral.com/submit

C Biomed Central 